\title{
Herencia medieval y neologismo en el léxico cromático de textos historiográficos en el siglo de Oro según el Corpus diacrónico del español
}

\section{María-Teresa Cáceres-Lorenzo ${ }^{1}$ D}

Accepted: 31 May 2021 / Published online: 25 October 2021

(C) The Author(s) 2021

\begin{abstract}
The main objective of this investigation is to examine with textual support the use of the vocabulary of color during the Golden Period through the investigation that it a medieval heritage or a neologism. This chromatic terminology was little used during the thirteenth century and in the following centuries its use is multiplied by the communication needs of a society that demands descriptive information on many social, cultural and economic issues, as happens with historiographic documents. To this end, a quantitative investigation has been designed in three phases: (a) search for chromatic voices that designate six selected colors; (b) determination of the first documentation of this group of voices; and (c) quantitative analysis to find a trend regarding the silver duality. For the search of these empirical references, the Diachronic Corpus of Spanish has been used as a data bank to collect textual examples on the chromatic vocabulary extracted from different historiographic sources. The result is the presentation of 100 terms with their respective empirical testimonies that reflect the continuity of the medieval heritage in the colors white, black and red, while formal neologisms are very frequent for the rest.
\end{abstract}

Keywords Chromatic vocabulary $\cdot$ Spanish historiographical texts $\cdot$ Neology Golden Age · Diachronic Corpus of Spanish

\section{Introducción}

El léxico cromático fue utilizado de manera parca en el periodo medieval con respecto a las siguientes centurias (Lapesa 2000: 268) tal como evidencia Duncan (1968 y 1975) al reunir cincuenta adjetivos de color del expurgo documental de unas 24 obras medievales. Algo similar aporta Martinell (1986: 133) quien analiza

María-Teresa Cáceres-Lorenzo

mteresa.caceres@ulpgc.es

1 Universidad de Las Palmas de Gran Canaria, Islas Canarias, Spain 
la expresión linguiística cromática en el Lapidario de Alfonso X (1250) y registra una docena de términos cromáticos: amarillo, azul, bermejo, baço, blanco, cárdeno, celeste, citrina, jalde, negro, pardo, verde, y zarco. El monarca castellano considera estos términos insuficientes para denominar toda la realidad cromática que se pretende definir, por lo que en su prosa se evidencias distintas estrategias para crear nuevas palabras, por ejemplo, procedentes del nombre de líquidos (aceite, sangre), metales (almagre), minerales (esmeralda, greda) y vegetales (azafrán, rosa), al mismo tiempo que rescata ejemplos procedentes de otras lenguas para designar una cualidad cromática.

Sánchez-Prieto Borja (2015: 26) al estudiar el glosario de la General Estoria dice que la tipología historiográfica es menos receptiva a los vocablos latinos con respecto a los textos científicos, a pesar de esto, constata su uso según lo siguiente, la voz nueva cuando aparece se explica o se acompaña mediante doblete junto a una patrimonial, y se informa al lector de que un determinado neologismo es extraño al castellano. Este procedimiento lingüístico continúa en el tiempo. Los siglos áureos representan una fase con grandes cambios en la evolución diacrónica del idioma español en cuanto al léxico (Verdonk y Mancho Duque 2010). Su crecimiento se articuló con frecuencia a través de estas estrategias medievales, además de con los préstamos de otras lenguas vivas o muertas, de la acuñación de nuevas palabras a través de sus diversos recursos derivados y de la incorporación de numerosos neologismos léxico-semánticos (Carriazo Ruiz 2015; Duncan 1975; González Sopeña 2019; Verdonk 2004).

Es decir, el catálogo de palabras del color en el español moderno, como otros lexicones, se incrementa por medio de la composición, derivación y parasíntesis tal como concluye García Macho (2010) al examinar el léxico de la navegación en el proyecto Diccionario de la Ciencia y de la Técnica del Renacimiento (DICTER). Sus conclusiones indican que muchos neologismos se originan más frecuentemente por medio de procesos derivativos que por préstamos léxicos. La denominación de la cualidad cromática de un objeto forma parte de la necesidad comunicativa de informar de manera descriptiva de una realidad nueva (peninsular o americana), pero realmente no sabemos cómo fue desde el punto de vista numérico ese incremento según las referencias textuales de las que disponemos en las bases de datos digitales, ni cuál es la tendencia seguida en los textos historiográficos, $\mathrm{y}$, sobre todo, si en la transición del bajo medievo al español moderno estamos ante un movimiento de creación de nuevos términos o los ejemplos pertenecen a periodos anteriores. Este planteamiento dual de herencia/innovación ya ha sido analizado para otras unidades léxicas de distintas profesiones o gremios por Mancho Duque (2005: 256). Esta investigadora aduce que el aumento del neologismo es ciertamente una predisposición en los siglos XVI y XVII, y estos no son vocablos cultos (aquellos que son entendidos solo por intelectuales o forman parte de un documento literario o científico), sino al contrario parecen favorecer la difusión y popularización de la información que se busca transmitir. Al mismo tiempo, Verdonk (2004: 896) dice que estas unidades léxicas "ya estaban atestiguadas en el siglo XV o incluso antes". 
El propósito general de esta investigación ${ }^{1}$ es mostrar datos empíricos del uso de los nombres de ciertos colores: amarillo, azul, blanco, negro, rojo y verde en textos historiográficos auriseculares. El lexicón que se recopile será útil para indagar sobre el crecimiento de este vocabulario a través del dato del primer registro textual antes o después del siglo XV, y con el seguimiento desde el punto de vista formal de los principales medios de renovación, préstamos y la construcción de nuevas unidades léxicas. Los documentos historiográficos constituyen un universo discursivo que comparten la finalidad comunicativa de narrar y dar a conocer acontecimientos dignos de ser recordados, por lo que el vocabulario tendrá la función de describir o transmitir información de manera clara y precisa tal como aparece en las cartas, crónicas, libro de viajes, relaciones en el que aparezcan los testimonios personales; memorias y diarios; etc. Las primeras relaciones solicitadas por Felipe II, que luego evolucionaron a textos más completos redactados con la guía de un largo cuestionario, podían ser "una narración breve y generalmente anónima sobre hechos variados: bélicos, políticos, biográficos, etc.” lo que conlleva un gran número de temas (Arroyo Ilera 2018: 172). A pesar de la necesidad de la terminología cromática en esta tipología textual, indagaciones precedentes no la han tenido en cuenta en este tipo de documentos (Dworkin 2016). Para recopilar testimonios documentales en un mayor número de textos manejaremos el Corpus Diacrónico del Español (CORDE) ya que este banco de datos reúne un número significativo de documentos del siglo de Oro que esta base de datos sitúa entre 1493 y 1713, además de permitir recuperar los ejemplos según los filtros del año y tipología textual necesarios para esta investigación.

El objetivo comunicativo de hacer presente o narrar algo con claridad, de manera minuciosa y sin omitir ninguna particularidad, por medio del lenguaje de los documentos historiográficos, es compartido en parte por otras tipologías como la científica o la jurídica. La historiografía no tiene por qué ser objetiva, lo que permite la presencia de la subjetividad, a pesar de que, con frecuencia, los autores indiquen que son testigos veraces de lo que escriben. Esta idea es desarrollada por Oesterreicher (1994: 157) para la historiografía indiana al demostrar que estos textos son producciones de escritores no siempre profesionales ni cultos y de diversa procedencia, esto es "oficiales con sus secretarios y escribanos, funcionarios, historiadores humanistas o cronistas de convento", lo que hace factible una clara tendencia hacia la variación y la comunicación más próxima a la oralidad. De igual forma, Bravo García y Cáceres Lorenzo (2013: 8) deducen que los distintos escritores de los textos históricos americanos a imitación de los peninsulares, ofrecen la información con gran detalle desde sus respectivos intereses particulares y diferentes grados de formación humanística. Es lo que Brendecke (2016: 40) indicó al afirmar que los testigos al servicio del la Corona rivalizan en presentar un conocimiento administrativo

\footnotetext{
${ }^{1}$ Esta investigación forma parte del Proyecto "Americanismos léxicos en las lenguas española e inglesa documentados en textos sobre América anteriores a 1700: AMERLEX-DATABASE" (PID2019104199 GB-I00) incluido en los Programas Estatales de Generación de Conocimiento y Fortalecimiento Científico y Tecnológico del Sistema de I+D +i del Ministerio de Ciencia e Innovación (Gobierno de España).
} 
y novedoso, relativo a la experiencia de los territorios del imperio español, lo que impulsa una descripción lo más completa posible. De hecho, en los textos se constata la urgencia de los poderes fácticos de recibir detalles minuciosos sobre determinados puntos geográficos como este fragmento del rey de España solicitando informes sobre el reino de Toledo (España peninsular):

El Rey. Nuestro alcalde mayor del partido de Zurita de la Orden de Calatrava. Por haber entendido que hasta agora no se ha hecho ni hay descripcion particular de los pueblos de estos reinos cual conviene a la autoridad e grandeza de ellos, habemos acordado que se haga dicha discrepcion y una historia de las particularidades y cosas notables de los dichos pueblos, y porque si se hubiesen de inviar personas a traer las relaciones que para ello son menester, no podría haber la brevedad con que holgariamos que esto se hiciese, ha parescido que por medio de los perlados e corregidores e justicias principales se podria hacer muy cumplidamente [...] y en que nos aviseis de como lo hobieredes ordenado e proveido, nos servireis. Del Pardo, a veinte e siete de otubre de mil e quinientos e setenta e cinco años. Yo el Rey. Por mandado de Su Magestad, Juan Vazquez. (CORDE, Anónimo, 1575-1580 Borrar 1535-c. 1552, Relaciones histórico-geográficas-estadísticas de los pueblos de España) [la negrita es nuestra]

La preocupación por contar con un testimonio minucioso a la vez que suscitar emoción patriótica no era una acción totalmente novedosa en el siglo XVI. Se trata de una práctica transmitida por la experiencia de los monarcas católicos en el Mediterráneo occidental, quienes reclaman detalles de la realidad para la explotación económica o con fines propagandísticos. En este texto historiográfico sobre el reino de Granada se comprueba la utilidad representativa de los colores de los bandos:

Miguel Acis, con la gente de las parroquias de San Gregorio, San Cristóbal y San Nicolás, á la puerta de Frex el Leuz, que cae en lo mas alto del Albaicin á la parte del cierzo, con una bandera ó estandarte de damasco carmesí con lunas de plata y fluecos de oro, que tenia hecha en su casa y guardada para aquel efeto; Diego Nigueli el mozo, con la gente de San Salvador, Santa Isabel de los Abades y San Luis, y una bandera de tafetan amarillo, á la plaza Bib el Bonut; y Miguel Mozagaz, con la gente de San Miguel, San Juan de los Reyes, y San Pedro y San Pablo, y una bandera de damasco turquesado, á la puerta de Guadix. (CORDE, Luis de Mármol Carvajal, 1600, Historia del Rebelion y Castigo de los Moriscos del Reyno de Granada) [la negrita es de nuestra]

Ante la necesidad de información particular de cada pueblo o lugar surge la condición del testigo acreditado para elaborar un texto historiográfico de manera que sea difundido (Collard 2008), pero, sobre todo, haciendo posible su comprensión a través de "la vivacidad y presencia sensorial que debían evocar las descripciones" (Carrillo Castillo 2004: 204) tal como aparece en muchos autores. Gansen (2019) añade a modo de ejemplo que el cronista Fernández de Oviedo fue pionero en indicar la importancia del color para describir la realidad americana, sobre la que escribe constituyendo un modelo para otros. Su esfuerzo por 
convertirse en un cronista al servicio del imperio español se materializaba en un texto, en el que se daba a conocer al público un determinado tema con muchos detalles que avalen su condición de experto. Fernández de Oviedo llegar a comparar su oficio con el de Leonardo Da Vinci:

Dezía Leonardo de Avinçi, Leonardo de Avinçi pintor exçelente que yo conoscí en casa del Duque de Milán Ludouico (que perdió aquel estado), que la exçelençia del pintor consistía, demás de entender muy bien su arte e medidas, que fuesen de las que muchos las deseasen, e quél desease que sus cosas pintadas paresçiesen de mucho rrelieue. E questo no podrá ser sin saber vsar bien de los colores (CORDE, Gonzalo Fernández de Oviedo, 1535 - c 1552, Batallas y quinquagenas)

Después de Fernández de Oviedo distintos escritores y temáticas desarrollan esta estrategia de construcción de un texto historiográfico en un contexto cultural barroco que concede una gran importancia a la visualidad (Goldberg 1992; González Ollé 1981; Vega García-Luengos 2013). A modo de ejemplo, López de Gómara escribe sobre los hombres de América, y aunque nunca salió de territorio de la España peninsular, considera útil la diferenciación de los colores del mestizaje (Alvar 1987; Stephens 1999). Este texto puede considerase un documento inicial sobre el léxico cromático aplicado posteriormente a diferentes grupos humanos:

Porque ay hombres blancos de muchas maneras de blancura, y bermejos de muchas maneras de bermejura, y negros de muchas maneras de negrura; $y$ de blanco va a bermejo por descolorido y ruvio, y a negro por ceniçoso, moreno, loro y leonado, como nuestros indios, los quales son todos en general como leonados o membrillos cochos, o tiriciados o castaños, y esta color es por naturaleza, y no por desnudez, como pensavan muchos, aunque algo les ayuda para ello yr desnudos, de suerte que assí como en Europa son comúnmente blancos y en África negros, assí también son leonados en nuestras Indias, donde tanto se maravillan de ver hombres blancos como negros. Es también de considerar que son blancos en Sevilla, negros en el cabo de Buena Esperança y castaños en el río de La Plata, estando en yguales grados de la equinocial; y que los hombres de África y de Asia que biven so la tórrida zona sean negros, y no lo sean los que biven debaxo la mesma zona en México, Yucatán, Quauhtemallán, [...] (CORDE, Francisco López de Gómara, 1554, La primera parte de la Historia natural de las Indias) [la negrita es nuestra]

Asimismo, un examen anticipado en los textos historiográficos del CORDE indica la urgencia de contar con un catálogo de palabras del color que se va incrementando durante esta etapa en distintas zonas geográficas, es lo que Coseriu (1992: 29) señala para el concepto de lengua histórica. En el este periodo áureo, el romance castellano se convierte en lengua histórica, en la que las nuevas necesidades comunicativas se manifiestan con soluciones que configuran la variedad y unidad lingüística. Las investigaciones sobre el vocabulario del color en el español áureo han tenido en cuenta el esquema propuesto por Berlin y Kay (1969) 
para definir la evolución diacrónica de los términos amarillo, azul, blanco, gris marrón, morado, naranja, negro, rojo, rosa y verde que designan el color y explicar cómo cada idioma va adquiriendo términos cromáticos en una secuencia estereotipada (focal colors). También los estudiosos se han centrado en los aspectos semánticos de listado de vocablos y preferencias en la descripción de la naturaleza (Conway et al. 2020; Peñas-Ibáñez 2017).

Espejo Muriel (1990 y 1996) analiza las preferencias que se extraen del estudio textual de 365 denominaciones cromáticas sobre la naturaleza recogidas de repertorios lexicográficos académicos desde el siglo XVIII, y llega a la conclusión de que el rojo con sus respectivas unidades léxicas se sitúa en primer lugar, seguido del negro, blanco, amarillo, verde y azul, por este orden. Esta misma escala de vitalidad es corroborada por Stala (2011), después del análisis de un lexicón de 135 palabras extraídas de obras literarias y lexicográficas de los siglos XVI y XVII. Finalmente, Jiménez Ríos (2016: 714) aporta datos cuantitativos sobre la presencia de adjetivos o participios neológicos obtenidos de 74 textos científicos del DICTER y afirma que un porcentaje significativo de estas unidades cromáticas, adjetivos o participios, aparecen en pocos casos; que las creaciones neológicas se construyen a través del léxico de la lengua general y de manera minoritaria con préstamos.

A tenor de lo anterior, se constata que la bibliografía precedente no ha tenido en cuenta el análisis del vocabulario cromático en documentos historiográficos de la etapa aurisecular. Tampoco se ha resuelto con casos empíricos la cuestión de que este catálogo de palabras proviene de periodo medieval o son vocablos creados en la España de los Austrias, por lo que planteamos las siguientes preguntas de investigación con la búsqueda en el CORDE entre 1493 y 1713: (a) ¿qué clasificación muestran las distintas unidades designativas cromáticas para referenciar los colores amarillo, azul, blanco, negro, rojo y verde con respecto al número de unidades léxicas que agrupa y de casos?; (b) ¿cómo se evidencia la neología formal? y finalmente, (c) ¿cuál es la tendencia que se obtiene sobre la caracterización del léxico del color como herencia medieval o neologismo, según el examen de la primera documentación de cada unidad léxica encontrada?

Esperamos que los resultados obtenidos sean una contribución a la evolución del léxico cromático como parte la transición léxica en el español del periodo medieval al áureo. Asimismo, se busca recopilar datos empíricos que puedan sumarse a otros sobre el uso del vocabulario en distintas tipologías textuales.

\section{Material y método}

La exploración cuantitativa se ha diseñado para dar respuestas a las preguntas de investigación y se estructura de la siguiente forma:

a) Recopilación de un glosario cromático obtenido de Duncan (1968); Martinell (1986); Espejo Muriel (1990 y 1996) y Stalan (2011), además de los que registra el Diccionario de Autoridades (DA). Este lexicón inicial de más de 400 palabras está formado por distintas unidades léxicas y sus correspondientes variantes gráficas que se agrupaban en torno a amarillo, azul, blanco, negro, rojo y verde. Asimismo, se ha utilizado el Nuevo Tesoro lexicográfico de la Real Academia Española (NTLLE) 
que permite consultar los principales diccionarios de los siglos XV al XVIII para reconocer el color al que se refieren las distintas denominaciones.

b) Búsqueda en el CORDE de este vocabulario cromático según las fechas que esta base de datos considera periodo áureo (1493-1713) y que constituye el 28\% de todos los documentos que reúne. Somos conscientes de que el CORDE tiene limitaciones como base de datos porque presenta ciertos problemas en la indagación. Esto se ha superado con la búsqueda de distintas grafías de una misma unidad léxica. Nuestro interés se focaliza en el subcorpus denominado "Historia y documentos" que representa el $24,17 \%$ de las 91.167 .616 palabras registradas en el CORDE. Dicha pesquisa ha hecho posible la determinación del periodo o centuria de la primera documentación de cada vocablo cromático obtenido.

c) Análisis cuantitativo de los resultados, conforme a lo propuesto en las preguntas de investigación.

\section{Análisis de los resultados}

Las muestras textuales obtenidas presentan 100 denominaciones cromáticas (principalmente, adjetivos, y sustantivos) que se agrupan con la consulta al NTLLE en las unidades léxicas amarillo, azul, blanco, negro, rojo y verde según aparecen en las Tablas 1 and 2. Nuestro inventario de búsqueda lo componían 400 unidades léxicas, por lo que solo tenemos noticias de un $25 \%$. La suma del número de términos indica que el rojo posee un mayor guarismo, y posteriormente, negro, blanco, amarillo, verde y azul, por lo que se confirma la clasificación establecida por Espejo Muriel (1990 y 1996) y Stalan (2011) para las referencias agrupadas por colores. Al mismo tiempo, los datos revelan que esta ordenación es distinta si tenemos en cuanta el factor del número de casos que proporciona el CORDE para 1493-1713, en que las designaciones del color negro con 3.074 casos y blanco con 2.236 ejemplos son mayoritarias, después el rojo con 1.611 casos y el amarillo con 1.060 ejemplos. Siguiendo con el análisis de las Tablas 1 and 2 llaman la atención los 58 vocablos de 100 recopilados que, en más de dos centurias, se registran en un número reducido de textos (10 o menos ocasiones). Este resultado corrobora lo expuesto por Jiménez Ríos con un número significativo de casos. De igual forma se constata la preferencia por ciertos términos con más de 500 ejemplos: amarillo, blanco, etc.

El préstamo de otras lenguas se evidencia en la procedencia latina de más del $70 \%$ de los términos del amarillo, verde y negro se complementa con un guarismo inferior en el blanco y rojo con 56,25\% y 53,57\% respectivamente. No sucede lo mismo con el azul, con un $28,57 \%$ de términos de procedencia latina. El aporte árabe con porcentajes superiores al $50 \%$ es significativo en el rojo y el azul tal como apuntaba González Sopeña (2019). Los ejemplos de los préstamos del francés o italiano aparecen minoritariamente entre 1 y 4 casos: acanelado 'semejante a la canela', ampo 'blanco luminoso', carmín, brunete 'negro’, jalde ‘amarillo subido' y turquesado `azul turquí'. Lo más peculiar es la presencia del indigenismo del Caribe embijado 'de color bermellón` que tiene difusión en áreas americanas del Cono Sur conforme los datos del CORDE. 
Table 1 Vocabulario del color en el CORDE en textos historiográficos

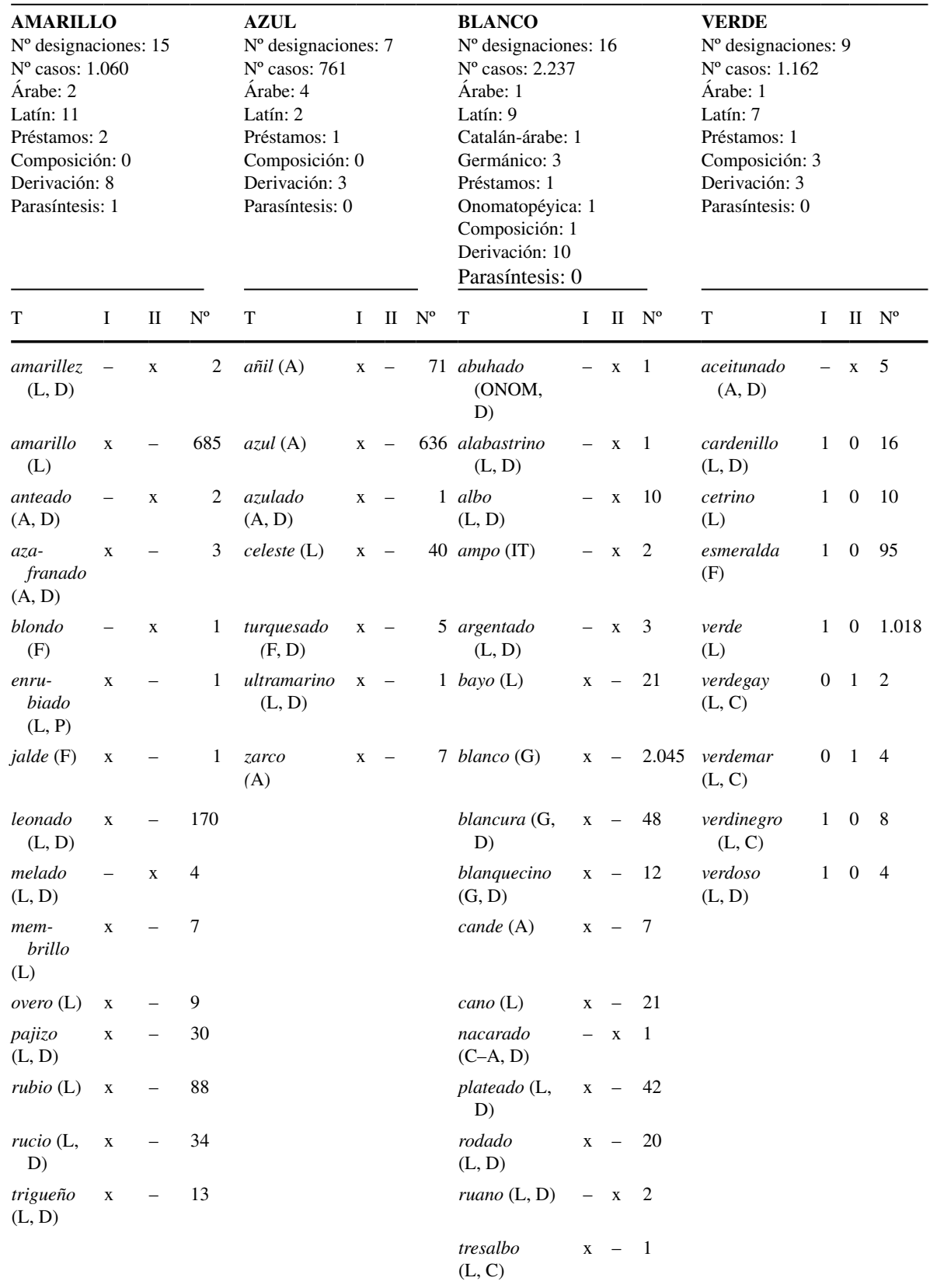

T: término cromático; I: 1493-1600; II: 1601-1713; No : numero de casos. Étimo: A: árabe; F: francés; C: catalán; G: Germánico; IT: italiano; L: latín; ONOM: onomatopéyica. Neologismo formal: C: composición; D: derivación; P: parasíntesis 
Iba el Francisco Martín en todo a la usanza de los indios, como hemos dicho. Vivía entre ellos desnudo en carnes y éstas ya percudidas y tostadas del sol, todo el cuerpo embijado, emplumada la cabeza, con su arco y flechas, el cabello largo, la barba y las demás partes de su cuerpo sin ningún pelo, las partes de la puridad cubiertas con un calabacillo pequeño, y al fin, en todo tan al natural indio, que era menester mirar por brújula para conocer que no lo era. (CORDE, Fray Pedro Simón, 1627, Primera parte de noticias historiales de las conquistas de tierra firme en las Indias Occidentales) [la negrita es nuestra]

[...] tuvo en el camino, por ir siempre á la ribera, muchos recuentros con los indios, que algunos dellos tienen esta calidad: cuando quieren que nadie entre en su tierra, so pena de la vida, toman un calabazo grande, y pasado con dos flechas ó tres y muy embijado, cuélganlo de un árbol; cuando no quieren hacer mal á los que entran en su tierra cuelgan una garza blanca, muerta, de un árbol. No es mal aviso para los comarcanos. (CORDE, Fray Reginaldo Lizárraga, 1605, Descripción breve de toda la tierra del Perú, Tucumán, Río de la Plata y Chile) [la negrita es nuestra]

La neología formal se produce con la construcción de nuevas unidades léxicas mediante la composición, derivación y parasíntesis, tal como evidencian las 32 nuevas lexías que derivan de sustantivos: acanelado de canela; aceitunado de aceituna; amulatado de mulato; azafranado de azafrán; cervuno de ciervo; noguerado de nogal; pajizo de paja; etc. Igualmente, berroqueña, granate y turquesado de las piedras que llevan estos nombres. Los mismos adjetivos crean otros para designar las diferencias: azulado, enrubiar, verdoso, etc.

El examen de las Tablas 1 and 2 ratifica otras conclusiones del marco teórico, como sucede con los datos en las designaciones del 'negro' que justifican lo dicho sobre la preferencia de negro en lugar de prieto con lo que se confirma lo expuesto por Dworkin (2016) que prueba la prevalencia de este color, aunque parece que prieto aparece preferentemente en textos americanos. En muchos ejemplos se cumple la definición del DA: "adj. que se aplica al color mui obscúro y que casi no se distingue del negro. Tómase muchas veces por el mismo color negro." Los párrafos de Díaz del Castillo y Sarmiento de Gamboa con una referencia al rojo del almagre en lugar de embijado:

Y estando en las estancias y maízales por mí ya dichas, tomando nuestra agua, vinieron por la costa muchos escuadrones de indios del pueblo de Potonchan (que así se dice), con sus armas de algodón que les daba a la rodilla, y con arcos y flechas, y lanzas y rodelas, y espadas hechas a manera de montantes de a dos manos, y hondas y piedras, y con sus penachos de los que ellos suelen usar, y las caras pintadas de blanco y prieto enalmagrados. (CORDE, Bernal Díaz del Castillo, 1568-1575, Historia verdadera de la conquista de la Nueva España) [la negrita es nuestra]

Viniendo una carabela de la isla de San Miguel a la isla de San Jorge a 15 de junio de este año de 1580, estando la carabela [[a]] 10 leguas de San Jorge, que podía ser como media hora antes que se pusiese el sol, vieron los hombres que venían en ella en el cuerpo del sol un crucifijo grande, y en el pie del cruci- 
Table 2 Vocabulario del color en el CORDE en textos historiográficos

\begin{tabular}{|c|c|c|c|c|c|c|c|c|c|c|c|c|c|c|c|}
\hline NEGRO & & & & & & & & ROJO & & & & & & & \\
\hline $\mathrm{N}^{\circ}$ designacic & ones & $: 24$ & & & & & & $\mathrm{~N}^{\circ}$ designacic & ones & 28 & & & & & \\
\hline $\mathrm{N}^{\circ}$ casos: 3.0 & & & & & & & & $\mathrm{~N}^{\circ}$ casos: 1.6 & & & & & & & \\
\hline Árabe: 1 & & & & & & & & Árabe: 7 & & & & & & & \\
\hline Latín: 18 & & & & & & & & Latín: 16 & & & & & & & \\
\hline Préstamos: 2 & & & & & & & & Préstamos: 4 & & & & & & & \\
\hline Composición & & & & & & & & Occitano: 1 & & & & & & & \\
\hline Derivación: 5 & & & & & & & & Composición & & & & & & & \\
\hline Parasíntesis: & & & & & & & & Derivación: 1 & & & & & & & \\
\hline & & & & & & & & Parasíntesis: & & & & & & & \\
\hline $\mathrm{T}$ & I & II & $\mathrm{N}^{\circ}$ & $\mathrm{T}$ & I & II & $\mathrm{N}^{\circ}$ & $\mathrm{T}$ & I & II & $\mathrm{N}^{\circ}$ & $\mathrm{T}$ & I & II & $\mathrm{N}^{\circ}$ \\
\hline $\begin{array}{l}\text { abrasado } \\
(i ?, \mathrm{P})\end{array}$ & - & $\mathrm{x}$ & 4 & $\begin{array}{l}\text { morcillo } \\
\text { (L) }\end{array}$ & $\mathrm{x}$ & - & 16 & $\begin{array}{l}\text { acanelado } \\
(\mathrm{F}, \mathrm{P})\end{array}$ & - & $\mathrm{x}$ & 3 & $\begin{array}{l}\text { embijado } \\
\text { (CAR, D) }\end{array}$ & - & $\mathrm{x}$ & 4 \\
\hline $\begin{array}{l}\text { ahumado } \\
(\mathrm{L}, \mathrm{D})\end{array}$ & $\mathrm{x}$ & - & 2 & $\begin{array}{l}\text { moreno } \\
\text { (L) }\end{array}$ & $\mathrm{x}$ & - & 53 & $\begin{array}{l}\text { alazán } \\
\text { (A) }\end{array}$ & $\mathrm{x}$ & - & 6 & $\begin{array}{l}\text { encarnado } \\
(\mathrm{L}, \mathrm{P})\end{array}$ & $\mathrm{x}$ & - & 224 \\
\hline $\begin{array}{l}\text { amulatado } \\
(\mathrm{L}, \mathrm{P})\end{array}$ & - & $\mathrm{x}$ & 4 & $\begin{array}{l}\text { mulato } \\
(\mathrm{L}, \mathrm{D})\end{array}$ & $\mathrm{x}$ & - & 81 & $\begin{array}{l}\text { alheñado } \\
\text { (A, D) }\end{array}$ & $\mathrm{x}$ & - & 3 & $\operatorname{grana}(\mathrm{L})$ & $\mathrm{x}$ & - & 150 \\
\hline $\begin{array}{l}\text { atezado } \\
(\mathrm{L}, \mathrm{D})\end{array}$ & - & $\mathrm{x}$ & 7 & negro $(\mathrm{L})$ & $\mathrm{x}$ & - & 2.024 & $\begin{array}{l}\text { almagrado } \\
(\mathrm{A}, \mathrm{D})\end{array}$ & $\mathrm{x}$ & - & 9 & $\begin{array}{l}\text { granate } \\
\quad(\mathrm{OCC})\end{array}$ & - & $\mathrm{x}$ & 2 \\
\hline $\begin{array}{l}\text { azabachado } \\
(\mathrm{A}, \mathrm{D})\end{array}$ & - & $\mathrm{x}$ & 1 & $\begin{array}{l}\text { negrura } \\
(\mathrm{L}, \mathrm{D})\end{array}$ & $\mathrm{x}$ & - & 11 & aloque (A) & - & $\mathrm{x}$ & 3 & $\operatorname{laca}(\mathrm{A})$ & - & $\mathrm{x}$ & 1 \\
\hline $\begin{array}{l}\text { barbinegro } \\
(\mathrm{L}, \mathrm{C})\end{array}$ & - & $\mathrm{x}$ & 4 & oscuro $(\mathrm{L})$ & $\mathrm{x}$ & - & 381 & bermejo $(\mathrm{L})$ & $\mathrm{x}$ & - & 81 & $\begin{array}{l}\text { noguerado } \\
(\mathrm{L}, \mathrm{D})\end{array}$ & - & $\mathrm{x}$ & 2 \\
\hline bazo $(\mathrm{L})$ & $\mathrm{x}$ & - & 7 & pardo $(\mathrm{L})$ & $\mathrm{x}$ & - & 321 & $\begin{array}{l}\text { bermejura } \\
\quad(\mathrm{L}, \mathrm{D})\end{array}$ & $\mathrm{x}$ & - & 3 & $\begin{array}{c}\text { pavonazo } \\
(\mathrm{I}, \mathrm{D})\end{array}$ & $\mathrm{x}$ & - & 1 \\
\hline brunete $(\mathrm{F})$ & $\mathrm{x}$ & - & 1 & prieto $(\mathrm{L})$ & $\mathrm{x}$ & - & 62 & cárdeno $(\mathrm{L})$ & $\mathrm{x}$ & - & 1 & rojo $(\mathrm{L})$ & $\mathrm{x}$ & - & 209 \\
\hline $\begin{array}{l}\text { cenizoso } \\
(\mathrm{L}, \mathrm{D})\end{array}$ & $\mathrm{x}$ & - & 2 & sable $(\mathrm{F})$ & $\mathrm{x}$ & - & 12 & buriel (L) & - & $\mathrm{x}$ & 7 & $\begin{array}{l}\text { rosado }(\mathrm{L}, \\
\mathrm{D})\end{array}$ & $\mathrm{x}$ & - & 62 \\
\hline $\begin{array}{c}\text { ceniciento } \\
(\mathrm{L}, \mathrm{D})\end{array}$ & $\mathrm{x}$ & - & 53 & $\begin{array}{r}\text { tapetado } \\
(i ?, \mathrm{D})\end{array}$ & - & $\mathrm{x}$ & 1 & carmesí (A) & $\mathrm{x}$ & - & 323 & $\begin{array}{l}\text { rosillo }(\mathrm{L}, \\
\mathrm{D})\end{array}$ & - & $\mathrm{x}$ & 2 \\
\hline $\begin{array}{c}\text { denegrido } \\
(\mathrm{L}, \mathrm{P})\end{array}$ & $\mathrm{x}$ & - & 10 & $\begin{array}{l}\text { tordillo }(\mathrm{L}, \\
\mathrm{D})\end{array}$ & & & & $\operatorname{carmin}(\mathrm{F})$ & - & $\mathrm{x}$ & 1 & $\begin{array}{l}\text { rubicundo } \\
\text { (L) }\end{array}$ & - & $\mathrm{x}$ & 1 \\
\hline fosco $(\mathrm{L})$ & $\mathrm{x}$ & - & 3 & & & & & castaño (L) & $\mathrm{x}$ & - & 60 & $\begin{array}{l}\text { sangriento } \\
(\mathrm{L}, \mathrm{D})\end{array}$ & $\mathrm{x}$ & - & 6 \\
\hline guineo & - & $\mathrm{x}$ & 3 & & & & & $\begin{array}{l}\text { cervuno } \\
(\mathrm{L}, \mathrm{D})\end{array}$ & - & $\mathrm{x}$ & 1 & $\begin{array}{l}\text { sanguino } \\
(\mathrm{L}, \mathrm{D})\end{array}$ & $\mathrm{x}$ & - & 22 \\
\hline loro $(\mathrm{L})$ & $\mathrm{x}$ & - & 5 & & & & & $\begin{array}{l}\text { colorado } \\
\text { (L) }\end{array}$ & $\mathrm{x}$ & - & 421 & zaino (A) & $\mathrm{x}$ & - & 3 \\
\hline
\end{tabular}

T: término cromático; I: 1493-1600; II: 1601-1713; No: numero de casos. Étimo: ¿?: desconocido o discutido; A: árabe; CAR: Caribe; F: francés; C: catalán; G: Germánico; IT: italiano; L: latín; OCC: occitano. Neologismo formal: C: composición; D: derivación; P: parasíntesis

fijo [[a]]parecía un calvario como suele pintarse, y vieron estar dos imágenes, una a la mano derecha vestida de blanco, y otra a la mano izquierda vestida, al parecer, medio de colorado, o como prieto. (CORDE, Pedro Sarmiento de Gamboa, 1580-1590, Los viajes al estrecho de Magallanes) [la negrita es nuestra]

La importancia de este léxico aparece en el Diccionario de Covarrubias (1611) al incorporar esta información en la voz bazo `[...] entre pardillo y negro, qual la 
suelen tener los mestizos, hijos de blanco y negra' (NTLLE) y el DA al citar una referencia a la crónica de Acosta en el lema atezado. Los ejemplos se registran no solo en América, sino también en Filipinas:

[...] la gente que en ellos venia era de color negro atezado y algunos más loros; hombres todos, de cabellos frisados, y muchos los traian blancos, rubios y de otros colores, por ser cierto el teñirlos y quitado la mitad del en la cabeza, y hechas otras diferencias; los dientes teñidos de colorado: venian todos desnudos, salvo partes que las cubrian con unas telas blondas, y con tinta más negra que su color embijados todos, y de otros colores hechas en el rostro y cuerpo algunas rayas. (CORDE, Anónimo, c. 1605-1609, Historia del descubrimiento de las regiones austriales hecho por el general Pedro Fernández de Quiró) [la negrita es nuestra]

En el CORDE no hemos encontrado ejemplos de payo como 'albino'; abrasilado, aciguatado o achocolatado para referirse al color negro de las personas por lo que no se ha podido corroborar lo afirmado por Alvar (1987) y Stephens (1999).

Los numerosos ejemplos de colorado (a) conviven con carmín procedente del francés con un único caso en documentos historiográficos (b) y frecuente en las tipologías del verso lírico y obras literarias (c), y carmesí con mayor número de casos en la prosa.

(a) Después desto llegó el arçobispo de Toledo vestido de damasco carmesí. $Y$ el autor dixo que este arçobispo, porque yva vestido de colorado, pareçía zebratana sangrienta (CORDE, Francés de Zúñiga, 1525 - 1529, Crónica burlesca del emperador Carlos $V$ ) [la negrita es nuestra]

(b) Quedó avergonçada la hermana de oír pretensión tan fea a un Sacerdote, y hermano suyo, a quien tenía en lugar de padre; y baxando con modestia virgen los ojos, remitió por entonces la respuesta al silencio, y al encendido carmín de su rostro; considerava los graves inconvenientes de qualquiera respuesta que diesse. (CORDE, Bernardo de Torres, 1657, Crónica agustina) [la negrita es nuestra]

(c) Esto pronuncia, y resuelto

del derecho lado muda

la daga que, ya desnuda,

vio en gualda el carmín envuelto [...]

(CORDE, Tirso de Molina, 1632, El bandolero) [la negrita es nuestra]

De hecho, en algunos autores aparecen las predilecciones de los españoles por carmesí (d, e, f) y se considera patrimonial por herencia arábiga (g) con un mecanismo que recuerda a los utilizados por Alfonso X en General Estoria al referenciar si el adjetivo es de procedencia romance o no (Sánchez-Prieto Borja 2015):

(d) En estas tunas, que son coloradas, nace la grana, que en esta lengua se llama mocheztlí. Es cosa tenida en mucho precio porque es muy subido colorado; entre los españoles se llama carmesí. (CORDE, Motolinía, Fray Toribio de Benavente, 1536 - 1541, Historia de los Indios de la Nueva España) [la negrita es nuestra] 
(e) Los españoles la llaman carmesí por ser color muy subido, y es de mucho precio. (CORDE, Francisco López de Gómara, 1553, Segunda parte de la Crónica general de las Indias) [la negrita es nuestra]

(f) Nuchtli es el color de la grana, tan subido que los españoles le llaman carmesí; [...] (CORDE, Francisco Cervantes de Salazar, 1560, Crónica de la Nueva España) [la negrita es nuestra]

(g) Sevilla es en nuestro tiempo de las celebradas, ricas y populosas çiudades del mundo; concurren a ella mercaderes de todo poniente, especialmente del Nuevo Mundo que llamamos Indias, con oro, plata, piedras preciosas y esmeraldas, poco menores de las que maravillaba el antigüedad en tiempo de los reyes de Egipto; pero en gran abundancia cueros, açúcar y la hierva que sirbe en lugar de púrpura, o por usar de bocablo arábigo y común, carmesí: cochinilla los indios la llaman donde ella se cría. (CORDE, Diego Hurtado de Mendoza, 1569-1573, De la guerra de Granada) [la negrita es nuestra]

Para responder a la cuestión de cuál es la tendencia del léxico del color en la posible de dualidad herencia medieval o neologismo, se crea la Tabla 3 con la primera documentación de las unidades léxicas encontradas.

El análisis de la Tabla 3 proporciona datos de las denominaciones del rojo y azul con un $57,14 \%$ de herencia medieval y blanco y negro con un $48,8 \%$. No sucede lo mismo con las referencias cromáticas del amarillo con un porcentaje de $13,3 \%$ y el verde con un $33,3 \%$. Esto supone el seguimiento de una tradición designativa medieval, cuestión que ya fue puesta de evidencia por Duncan, Martinell y Sánchez-Prieto Borja. Un ejemplo muy claro de esta pervivencia lo encontramos en los nombres de los pelos de los caballos: alazán `rojo’; bayo `dorado bajo’; castaño; melado ‘color miel’; morcillo `negro’; overo `color del huevo'; rodado `blanco'; rosillo; rucio `pardo claro'; ruano `gris’; zaino 'color castaño'; tresalbo 'blanco en tres de las patas'. La caracterización cromática de los equinos es común por lo que debe ser reconocida por un gran número de lectores:

Quiero aquí poner por memoria todos los caballos y yeguas que pasaron. El capitán Cortés, un caballo castaño zaino, que luego se le murió en San Juan de Ulúa. Pedro de Alvarado y Hernando López de Avila, una yegua castaña muy buena, de juego y de carrera; y de que llegamos a la NuevaEspaña el Pedro de Alvarado le compró la mitad de la yegua, o se la tomó por fuerza. Alonso Hernández Puertocarrero, una yegua rucia de buena carrera, que le compró Cortés por las lazadas de oro. Juan Velázquez de León, otra yegua rucia muy poderosa, que llamábamos "la rabona", muy revuelta y de buena carrera. Cristóbal de Olí, un caballo castaño oscuro, harto bueno. Francisco de Montejo y Alonso de Avila, un caballo alazán tostado: no fue para cosa de guerra. Francisco de Morla, un caballo castaño oscuro, gran corredor y revuelto. Juan de Escalante, un caballo castaño claro, tresalvo; no fue bueno. Diego de Ordás, una yegua rucia, machorra, pasadera (CORDE, Bernal Díaz del Castillo, 1568-1571, Historia verdadera de la conquista de la Nueva España) [la negrita es nuestra] Llevaba un sayo de brocado y raso carmesí muy lucido, sobre un caballo ruano muy bueno y muy bien encubertado, y todo de la misma divisa, y delante de él hasta cincuenta alabarderos a pie de su guarda. Los cuales, 
al tiempo del romper, se metieron o recogieron en la infantería. (CORDE, Fray Prudencio de Sandoval, 1604-1618, Historia de la vida y hechos del Emperador Carlos $V$ ) [la negrita es nuestra]

Mirando el rey y los demás caballeros al caballero cristiano, le vieron pasearse sobre un hermoso caballo de color tordillo, los relinchos del cual muy claramente se oían en el Alhambra. (CORDE, Ginés Pérez de Hita, 1595, Guerras civiles de Granada) [la negrita es nuestra]

En los documentos consultados en el CORDE, los vocablos de colores relacionados con los conocimientos de los albéitares siempre se utilizan para referirse a los caballos, con la excepción de bayo que aparece en 1614 en la descripción de un guanaco 'mamífero propio de los Andes similar a la llama':

De caza gruesa de montería sólo hay guanacos, que habitan las faldas de la Cordillera Nevada. Son muy semejantes en la traza, figura y grandeza a las ciervas, y en que no crían como ellas cuernos. Sólo difieren en que tienen el pelo más lanudo por la parte superior, con manchas de color bayo y blanco: relinchan los machos como potrillos. (CORDE, Alonso González Nájera, 1614, Desengaño y reparo de la guerra del reino de Chile) [la negrita es nuestra]

En la Figura 1 se representa gráficamente la tendencia de cómo se han formado los 100 ejemplos del vocabulario cromáticos extraído del CORDE, y se aprecia la escasez de ejemplo para los colores verde y azul tal como concluyen Conway et al. (2020).

Las voces heredadas del medievo son numerosas, con 45 ejemplos, pero en el periodo 1493 a 1600 se fundamentan textualmente y por primera vez, un buen número de casos, 39 lexías que se distribuyen de manera distinta entre los colores seleccionados. Entre 1601 y 1713 se registran 16 ejemplos. En definitiva, en los primeros años se crearon nuevas terminologías cromáticas y en la última centuria la propensión no es tan prolija, al menos en los documentos historiográficos.

\section{Conclusiones}

A la luz del centenar de palabras cromáticas obtenidas y que representan un $25 \%$ de las que se buscaban se puede aseverar que son necesarias más indagaciones en otras tipologías. Es posible que la utilización del vocabulario cromático para narrar, describir y hacer visible la realidad de los pueblos de España y América tenga otros datos de acuerdo a la finalidad comunicativa de los textos. Los resultados de los nombres de los colores extraídos del CORDE mantienen la clasificación de otros autores (Muriel 1996; Stalan 2011), pero se añade otra ordenación creada conforme al número de casos recogidos que ayuda a comprender la evolución diacrónica de este vocabulario con datos textuales. Parece que se corrobora que los términos de un color aumentan si son útiles para la comunidad que los utiliza, esto no es nuevo ya que Alfonso X en el siglo XIII sigue la misma tendencia (Sánchez-Prieto Borja 2015). En lo que se refiere a los mecanismos de construcción de nuevas 
Table 3 Primera documentación en textos historiográficos según CORDE

$1^{a}$ DOCUMENTACIÓN DEL LÉXICO CROMÁTICO EN TEXTOS (EDAD MEDIA HASTA 1713)

\begin{tabular}{|c|c|c|c|c|c|c|c|c|c|c|c|c|c|c|c|c|c|}
\hline \multicolumn{3}{|c|}{$\begin{array}{l}\text { AMARILLO } \\
\text { EM: } 20 \% \\
\text { I: } \mathbf{6 0 \%} \\
\text { II: } 20 \%\end{array}$} & \multirow{2}{*}{\multicolumn{2}{|c|}{ II }} & \multicolumn{3}{|c|}{$\begin{array}{l}\text { AZUL } \\
\text { EM: } \mathbf{5 7 , 1 4 \%} \\
\text { I: } \mathbf{4 2 , 8 5 \%} \\
\text { II: } 0\end{array}$} & \multirow{2}{*}{\multicolumn{2}{|c|}{ II }} & \multicolumn{3}{|l|}{$\begin{array}{l}\text { BLANCO } \\
\text { EM: } \mathbf{5 0} \% \\
\text { I: } 43,7 \% \\
\text { II: } 6,25 \%\end{array}$} & \multirow[b]{2}{*}{ II } & \multicolumn{4}{|c|}{$\begin{array}{l}\text { VERDE } \\
\text { EM: } \mathbf{3 3 , 3 \%} \\
\text { I: } \mathbf{3 3 , 3 \%} \\
\text { II: } \mathbf{3 3 , 3 \%}\end{array}$} \\
\hline $\mathrm{T}$ & EM & I & & & $\mathrm{T}$ & EM & I & & & $\mathrm{T}$ & EM & I & & $\mathrm{T}$ & EM & I & II \\
\hline $\begin{array}{l}\text { ama- } \\
\text { rillez }\end{array}$ & - & - & & $\mathrm{x}$ & añil & - & $\mathrm{x}$ & & - & abuhado & - & $\mathrm{x}$ & - & $\begin{array}{r}\text { aceitu- } \\
\text { nado }\end{array}$ & - & - & $\mathrm{x}$ \\
\hline amarillo & $\mathrm{x}$ & - & & - & azul & $\mathrm{x}$ & - & & - & $\begin{array}{c}\text { alabas- } \\
\text { trino }\end{array}$ & - & $\mathrm{x}$ & - & $\begin{array}{r}\text { carde- } \\
\text { nillo }\end{array}$ & - & $\mathrm{x}$ & - \\
\hline anteado & - & - & & $\mathrm{x}$ & azulado & - & $\mathrm{x}$ & & - & albo & $\mathrm{x}$ & - & - & cetrino & $\mathrm{x}$ & - & - \\
\hline $\begin{array}{l}\text { aza- } \\
\quad \text { franado }\end{array}$ & - & $\mathrm{x}$ & & - & celeste & - & $\mathrm{x}$ & & - & атро & - & $\mathrm{x}$ & - & $\begin{array}{c}\text { esmer- } \\
\text { alda }\end{array}$ & $\mathrm{x}$ & - & - \\
\hline blondo & - & - & & $\mathrm{x}$ & $\begin{array}{c}\text { turque- } \\
\text { sado }\end{array}$ & $\mathrm{x}$ & - & & - & $\begin{array}{l}\text { argen- } \\
\text { tado }\end{array}$ & $\mathrm{x}$ & - & - & verde & $\mathrm{x}$ & - & - \\
\hline $\begin{array}{l}\text { enru- } \\
\text { biado }\end{array}$ & - & $\mathrm{x}$ & & - & $\begin{array}{l}\text { ultrama- } \\
\text { rino }\end{array}$ & $\mathrm{x}$ & - & & - & bayo & $\mathrm{x}$ & - & - & $\begin{array}{l}\text { verde- } \\
\text { gay }\end{array}$ & - & - & $\mathrm{x}$ \\
\hline jalde & $\mathrm{x}$ & - & & - & zarco & $\mathrm{x}$ & - & & - & blanco & $\mathrm{x}$ & - & - & $\begin{array}{l}\text { verde- } \\
\text { mar }\end{array}$ & - & - & $\mathrm{x}$ \\
\hline leonado & - & $\mathrm{x}$ & & - & & & & & & blancura & $\mathrm{x}$ & - & - & $\begin{array}{l}\text { ver- } \\
\text { dine- } \\
\text { gro }\end{array}$ & - & $\mathrm{x}$ & - \\
\hline melado & - & $\mathrm{x}$ & & - & & & & & & $\begin{array}{l}\text { blanque- } \\
\text { cino }\end{array}$ & - & $\mathrm{x}$ & - & verdoso & - & $\mathrm{x}$ & - \\
\hline $\begin{array}{l}\text { mem- } \\
\text { brillo }\end{array}$ & - & $\mathrm{x}$ & & - & & & & & & cande & - & $\mathrm{x}$ & - & & & & \\
\hline overo & - & $\mathrm{x}$ & & - & & & & & & cano & $\mathrm{x}$ & - & - & & & & \\
\hline pajizo & - & $\mathrm{x}$ & & - & & & & & & nacarado & - & - & $\mathrm{x}$ & & & & \\
\hline rubio & $\mathrm{x}$ & - & & - & & & & & & plateado & - & $\mathrm{x}$ & - & & & & \\
\hline rucio & - & $\mathrm{x}$ & & - & & & & & & rodado & $\mathrm{x}$ & - & - & & & & \\
\hline trigueño & - & $\mathrm{x}$ & & - & & & & & & ruano & $\mathrm{x}$ & - & - & & & & \\
\hline & & & & & & & & & & tresalbo & - & $\mathrm{x}$ & - & & & & \\
\hline $\begin{array}{l}\text { NEGRO } \\
\text { EM: } 46 \% \\
\text { I: } 33 \% \\
\text { II: } 21 \%\end{array}$ & & & & & & & & & & $\begin{array}{l}\text { ROJO } \\
\text { EM: } \mathbf{5 7 , 1} \\
\text { I: } \mathbf{2 8 , 5 7 \%} \\
\text { II: } \mathbf{1 4 , 2 8} \%\end{array}$ & & & & & & & \\
\hline $\mathrm{T}$ & $\mathrm{El}$ & & I & II & $\mathrm{T}$ & & $M$ & I & II & $\mathrm{T}$ & EM & I & II & $\mathrm{T}$ & EM & I & II \\
\hline abrasado & - & & - & $\mathrm{x}$ & morcillo & $x$ & & - & - & $\begin{array}{r}\text { acane- } \\
\text { lado }\end{array}$ & - & - & $\mathrm{x}$ & embijado & - & $\mathrm{x}$ & - \\
\hline ahumado & - & & $\mathrm{x}$ & - & moreno & $x$ & & - & - & alazán & $\mathrm{x}$ & - & - & $\begin{array}{l}\text { encar- } \\
\text { nado }\end{array}$ & $\mathrm{x}$ & - & - \\
\hline amulatado & - & & - & $\mathrm{x}$ & mulato & - & & $\mathrm{x}$ & - & alheñado & $\mathrm{x}$ & - & - & grana & $\mathrm{x}$ & - & - \\
\hline atezado & - & & $\mathrm{x}$ & - & negro & $x$ & & - & - & $\begin{array}{l}\text { alma- } \\
\text { grado }\end{array}$ & $\mathrm{x}$ & - & - & granate & - & $\mathrm{x}$ & - \\
\hline azabachado & - & & - & $\mathrm{x}$ & negrura & $x$ & & - & - & aloque & - & $\mathrm{x}$ & - & laca & - & - & $\mathrm{x}$ \\
\hline
\end{tabular}


Table 3 (continued)

\begin{tabular}{|c|c|c|c|c|c|c|c|c|c|c|c|c|c|c|c|}
\hline \multicolumn{6}{|l|}{$\begin{array}{l}\text { NEGRO } \\
\text { EM: } 46 \% \\
\text { I: } 33 \% \\
\text { II: } 21 \%\end{array}$} & \multirow{2}{*}{\multicolumn{2}{|c|}{ I }} & \multicolumn{8}{|c|}{$\begin{array}{l}\text { ROJO } \\
\text { EM: } \mathbf{5 7 , 1 4 \%} \\
\text { I: } \mathbf{2 8 , 5 7 \%} \\
\text { II: } \mathbf{1 4 , 2 8 \%}\end{array}$} \\
\hline$\overline{\mathrm{T}}$ & EM & I & II & $\mathrm{T}$ & EM & & & $\bar{T}$ & EM & I & II & $\mathrm{T}$ & EM & I & II \\
\hline barbinegro & - & - & $\mathrm{x}$ & oscuro & $\mathrm{x}$ & - & - & bermejo & $\mathrm{x}$ & - & - & $\begin{array}{c}\text { noguer- } \\
\text { ado }\end{array}$ & - & - & $\mathrm{x}$ \\
\hline bazo & - & $\mathrm{x}$ & - & pardo & $\mathrm{x}$ & - & - & $\begin{array}{c}\text { berme- } \\
\text { jura }\end{array}$ & $\mathrm{x}$ & - & - & pavonazo & - & $\mathrm{x}$ & - \\
\hline brunete & $\mathrm{x}$ & - & - & prieto & $\mathrm{x}$ & - & - & buriel & - & $\mathrm{x}$ & - & rojo & $\mathrm{x}$ & - & - \\
\hline cenizoso & $\mathrm{x}$ & - & - & sable & - & $\mathrm{x}$ & - & cárdeno & $\mathrm{x}$ & - & - & rosado & $\mathrm{x}$ & - & - \\
\hline ceniciento & - & $\mathrm{x}$ & - & tapetado & - & - & $\mathrm{x}$ & carmesí & $\mathrm{x}$ & - & - & rosillo & - & $\mathrm{x}$ & - \\
\hline denegrido & - & $\mathrm{x}$ & - & tordillo & - & $\mathrm{x}$ & - & carmín & - & $\mathrm{x}$ & - & $\begin{array}{l}\text { rubi- } \\
\text { cundo }\end{array}$ & - & $\mathrm{x}$ & - \\
\hline fosco & $\mathrm{x}$ & - & - & & & & & castaño & $\mathrm{x}$ & - & - & $\begin{array}{c}\text { sangri- } \\
\text { ento }\end{array}$ & $\mathrm{x}$ & - & - \\
\hline guineo & - & $\mathrm{x}$ & - & & & & & cervuno & $\mathrm{x}$ & - & - & sanguino & $\mathrm{x}$ & & - \\
\hline loro & $\mathrm{x}$ & - & - & & & & & colorado & $\mathrm{x}$ & - & - & zaino & - & - & $\mathrm{x}$ \\
\hline
\end{tabular}

T: término cromático; EM: Edad Media; I: 1493-1600; II: 1601-1713

voces cromáticas, la derivación presenta un uso considerable con respecto a la composición y parasíntesis, tal como explicaba García Macho (2010).

Con respecto a la dualidad herencia o neologismo, las denominaciones del color en los siglos áureos heredan cerca de un $45 \%$ del periodo medieval. En el periodo 1493-1600 se documenta casi un 39\% y el resto aparecen por primera vez entre 1601 y 1713. Pudiera decirse que los textos revelan un proceso parcial de revitalización de la herencia medieval en la designación del color que se inicia en los textos antes del XV, pero que continua de manera progresiva y ralentizada hasta el siglo XVIII si tenemos en cuenta las investigaciones de Espejo Muriel con más de 300 ejemplos. En este proceso continuado muchas designaciones del color (adjetivos, participios y sustantivos) se registran en pocos textos como indica Jiménez Ríos (2016) para los textos científicos, lo que debiera analizarse en siglos posteriores. Asimismo, se confirma, con González Ollé (1981) y Goldberg (1992), que otras designaciones cromáticas se utilizan de manera abundante porque son reconocidas por los lectores. Por su parte, Oesterreicher (1994: 157) explica la necesidad en los textos historiográficos de la comunicación comprensible para dar testimonio de una experiencia conocida por el autor, aunque sea un vocablo nuevo que se explica o acompaña de un doblete. El neologismo puede surgir para favorecer una mayor comprensión en los destinatarios (Mancho Duque 2005).

Y, por último, este trabajo con datos extraídos de documentos y analizados a la luz del marco teórico, supone una contribución metodológica de un estudio de caso al problema de investigación de la configuración del léxico aurisecular. Quedan pendientes los cambios de significado que se producen en las descripciones del mundo animal o vegetal usadas para designar a las personas, como también los ejemplos de distanciamiento semántico entre un préstamo y la voz patrimonial, o cómo se 


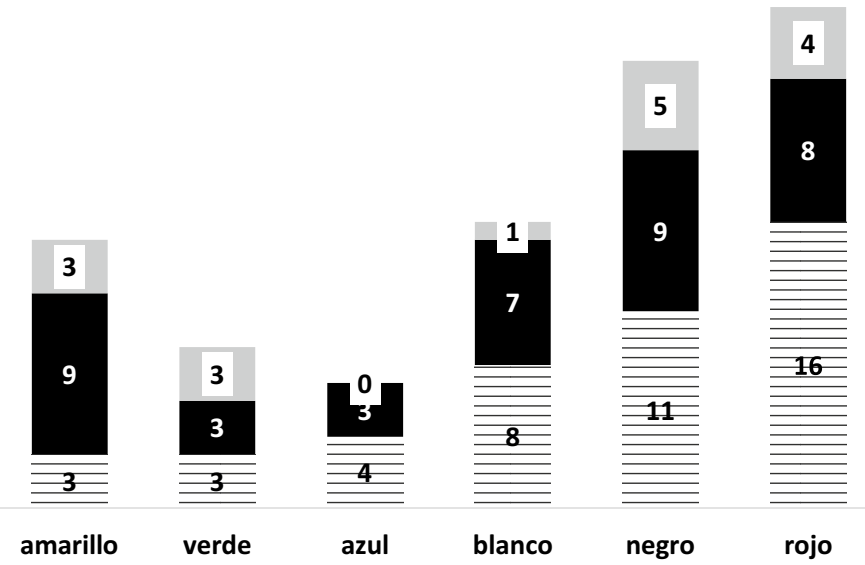

Fig. $1 \mathrm{~N}^{\mathrm{o}}$ de vocablos que se incrementan acorde a los datos de la primera documentación del CORDE

seleccionan unas designaciones frente a otras en distintas áreas geográficas. Futuros análisis podrán aportar otros ejemplos de esta tipología o de otras con el fin de obtener testimonios del proceso seguido por la lengua española con respecto a la designación del color. La suma de indagaciones es la única vía de reconstrucción documental de la historia del léxico.

Open Access This article is licensed under a Creative Commons Attribution 4.0 International License, which permits use, sharing, adaptation, distribution and reproduction in any medium or format, as long as you give appropriate credit to the original author(s) and the source, provide a link to the Creative Commons licence, and indicate if changes were made. The images or other third party material in this article are included in the article's Creative Commons licence, unless indicated otherwise in a credit line to the material. If material is not included in the article's Creative Commons licence and your intended use is not permitted by statutory regulation or exceeds the permitted use, you will need to obtain permission directly from the copyright holder. To view a copy of this licence, visit http://creativecommons.org/licen ses/by/4.0/.

\section{Referencias}

Alvar, M. (1987). Léxico del mestizaje en Hispanoamérica. Madrid: Cultura Hispánica D. L.

Arroyo Ilera, F. (2018). Las relaciones geográficas y el conocimiento del territorio en tiempos de Felipe II. Estudios Geográficos, 59, 169-200. https://doi.org/10.3989/egeogr.1998.i231.600.

Berlin, B. y Kay, P. (1969). Basic color terms: Their universality and evolution. Berkeley: University of California.

Bravo García, E. y Cáceres Lorenzo, M.-T. (2013). El léxico cotidiano en América a través de las Relaciones Geográficas de Indias. (Tierra firme y América del Sur, siglo XVI). Berna: Peter Lang.

Brendecke, A. (2016). Imperio e información funciones del saber en el dominio colonial español. Madrid: Iberoamericana. 
Carriazo Ruiz, J. R. (2015). El vocabulario de la navegación en el Siglo de Oro. Servizo de Publicacions: Universidade da Coruña.

Carrillo Castillo, J. (2004). Naturaleza e imperio: la representación del mundo natural en la 'Historia general y natural de las Indias' de Gonzalo Fernández de Oviedo. Madrid: Fundación Carolina.

Collard, P. (2008). Oro y hambre en la primera Década de Pedro Mártir de Anglería. Neophilologus, 92, 617-625. https://doi.org/10.1007/s11061-007-9069-5.

Conway, B., Ratnasingama, S., Jara-Ettingerb, J., Futrellc, R., y Gibson, E. (2020). Communication efficiency of color naming across languages provides a new framework for the evolution of color terms. Cognition, 195, 1-20. https://doi.org/10.1016/j.cognition.2019.104086.

Corpus Diacrónico del Español (CORDE). Real Academia Española: https://www.rae.es/recursos/bancode-datos/corde Consultado el 20 de marzo de 2020.

Coseriu, E. (1992). Lingüística histórica e historia de las lenguas. Boletín de Filología, 33(1), 27-33.

Diccionario de Autoridades (1726-1739) (DA). Real Academia Española: https:/www.rae.es/recursos/ banco-dedatos/corde. Consultado el 20 de marzo de 2020.

Duncan, M. (1968). Adjetivos de color en el español medieval. Anuario de Estudios Medievales, 5, 463-472.

Duncan, M. (1975). Color words in medieval Spanish. En S. Beardsley et al. (eds.), Studies in honor of Lloyd A. Kasten (págs. 53-71). Madison: Hispanic Seminary of Medieval Studies.

Dworkin, S. (2016). La estabilidad léxica: la pervivencia de los nombres de los colores del latín en español y en las otras lenguas romances. En A. López Serena, A. Narbona y S. del Rey Quesada (eds.), El español a través del tiempo: estudios ofrecidos a Rafael Cano Aguilar (págs. 245-257). Sevilla: Universidad.

Espejo Muriel, M. (1990). Los nombres de color en la naturaleza: estudio onomasiológico. Granada: Universidad.

Espejo Muriel, M. (1996). Los nombres de color en la naturaleza. Granada: Universidad.

Gansen, E. (2019). Framing the Indies: The Renaissance aesthetics of Gonzalo Fernández de Oviedo (1478-1557). Colonial Latin American Review, 28(2), 130-151. https://doi.org/10.1080/10609164. 2019.1627132 .

García Macho, M. L. (2010). El neologismo en el léxico de la navegación del Siglo de Oro. En L. Mancho Duque y R. Verdonk (eds.), Aspectos de la neología en el Siglo de Oro: lengua general y lenguajes especializados (págs. 111-130). Rodopi: Foro Hispánico. https://doi.org/10.1163/97890 42031180_008.

Goldberg, H. (1992). A reappraisal of colour symbolism in the courtly prose fiction of late-medieval Castile. Bulletin of Hispanic Studies, 69(3), 221-238. https://doi.org/10.1080/1475382922000369221.

González Ollé, F. (1981). Fisiognómica del color rojizo en la literatura española del Siglo de Oro. Revista de Literatura, 43/86 (1), 153-164.

González Sopeña, I. (2019). Arabismos vinculados a la industria textil del reino de Granada a través de sus documentos: aceituní, almaizar, alquicel, anafaya. Neophilologus,22, 23-44. https://doi.org/10. 1007/s11061-019-09633-6.

Jiménez Ríos, E. (2016). Neología y construcción del texto científico técnico en el siglo XVI: observaciones a propósito del uso de los adjetivos. Zeitschrift für romanische Philologie, 132-3, 711-730. https://doi.org/10.1515/zrp-2016-0048.

Lapesa, R. (2000). Historia de la lengua española. Madrid: Gredos.

Mancho Duque, M. J. (2005) La divulgación científica y sus repercusiones léxicas en la época de El Quijote. En Sánchez Ron, J. (ed.), La ciencia y El Quijote (págs. 245-257). Barcelona: Crítica.

Martinell, E. (1986). Expresión lingüística del color en el "Lapidario" de Alfonso X. Cahiers de linguistique hispanique médiévale, 11, 133-149.

Nuevo Tesoro lexicográfico de la Real Academia Española (NTLLE). Real Academia Española: https:// www.rae.es/recursos/diccionarios/diccionarios-anteriores-1726-1992/nuevo-tesoro-lexicografico. Consultado el 20 de marzo de 2020.

Oesterreicher, W. (1994). El español en textos escritos por semicultos. Competencia escrita de impronta oral en la historiografía indiana. En J. Lüdtke (ed.), El español de América en el siglo XVI (págs. 155-190). Madrid: Iberoamericana.

Peñas-Ibáñez, A. (2017). Denominación de las categorías de color básicas procesos ontogenéticos y semántico-cognitivos. RILCE: Revista de filología hispánica, 33, 1224-1267. https://doi.org/10. 15581/008.33.3.1224-67.

Sánchez-Prieto Borja, P. (2015). El léxico de la General Estoria de Alfonso X el Sabio. Anuario de Estudios Medievales, 45, 17-53. https://doi.org/10.3989/aem.2015.45.1.01. 
Stala, E. (2011). Los nombres de los colores en el español de los siglos XVI-XVII. Alicante: Biblioteca Virtual Miguel de Cervantes: http://www.cervantesvirtual.com/obra/los-nombres-de-los-colores-enel-espanol-de-los-siglos-xvi-xvii/. Consultado el 20 de marzo de 2020.

Stephens, T. (1999). Dictionary of Latin American racial and ethnic terminology. Florida: University of Florida Press.

Vega García-Luengos, G. (2013). Juegos y pasatiempos con colores en el teatro español del siglo XVII. Bulletin of Spanish Studies, 90(4/5), 845-870 https://doi.org/10.1080/14753820.2013.802597.

Verdonk, R. (2004). Cambios en el léxico del español durante la época de los Austrias. En R. Cano Aguilar (ed.), Historia de la lengua española (págs. 895-918). Barcelona: Crítica.

Verdonk, R. y Mancho Duque, M. J. (2010). Aspectos de la neología en el Siglo de Oro: lengua general y lenguajes especializados. Brill: Rodopi.

Publisher's Note Springer Nature remains neutral with regard to jurisdictional claims in published maps and institutional affiliations. 